\title{
Microwave pulse delay at propagation through the 1D electromagnetic crystals
}

\author{
V.S. Babitski ${ }^{1}$, V.G. Baryshevsky ${ }^{2}$, A.A. Gurinovich ${ }^{2}$, E.A. Gurnevich ${ }^{2}$, P.V. Molchanov ${ }^{2}$, \\ L.V. Simonchik ${ }^{1}$, M.S. Usachonak ${ }^{1}$ and R.F. Zuyeuski \\ ${ }^{1}$ Institute of Physics of NAS of Belarus, Minsk, Belarus, 1.simonchik@ifanbel.bas-net.by \\ ${ }^{2}$ Institute for Nuclear Problems of BSU, Minsk, Belarus \\ ${ }^{3}$ Museo Storico della Fisica e Centro di Studi e Ricerche "Enrico Fermi", Roma, Italy \& CERN
}

Interest to electromagnetic pulses propagation in matter with the group velocity much smaller than the speed of light in vacuum increases recent decades. Effect of group velocity reduction for a wave in a spatially periodic matter (natural crystal, photonic crystal, etc.) exists for different ranges and variety of waves (X-ray, optical, $\mathrm{THz}$ and microwave) due to generality of laws of wave diffraction. Multiple stu-dies report significant reduction of group velocity $v_{g r}$ of an X-ray pulse passing through a crystal, when Bragg diffraction occurs [1]. A neutron delay in crystal in conditions of diffraction was predicted and observed [2]. Slow light, which is a promising solution for time-domain processing of optical signals, is a subject for multiple studies [3]. Experimental observation of this effect for microwave radiation generated by an electron beam in the photonic crystal built of metal threads was reported in [4]. a)

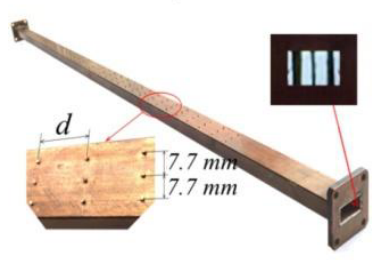

Fig. 1. Photonic crystal photo (a) and its frequency response (b) b)

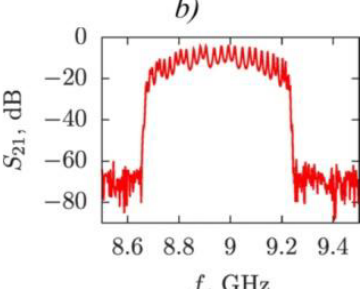

$f, \mathrm{GHz}$ waveguide corresponds to $\mathrm{TE}_{10}$ mode of a rectangular waveguide. Since the delay time is proportional to the length of photonic crystal, the number of periods $\mathrm{N}$ was chosen to be rather high $(\mathrm{N}=33)$ to make the delay time convenient for measuring. The photonic crystal frequency response is presented at Fig. 1, $b$ and shows some amplitude ripples, which number is determined by the number of crystal periods.

Microwave pulse propagation in the photonic crystal was simulated by CST Microwave Studio [5] to define the incident pulse width making pulse delay effect evident. The simulation of transmitted and reflected pulses in time-domain was produced at the incident pulse duration from 4 to $30 \mathrm{~ns}$. It was shown that delay effect is well resolved, when the width of the incident pulse is $10 \mathrm{~ns}$ and shorter. The time distance between maxima of transmitted and reflected pulses is about $23 \mathrm{~ns}$ (Fig. 2), that corresponds to pulse delay time $\tau$. Both the transmitted and reflected pulses have chain of equidistant peaks with time interval between adjacent those of about $2 \tau$.

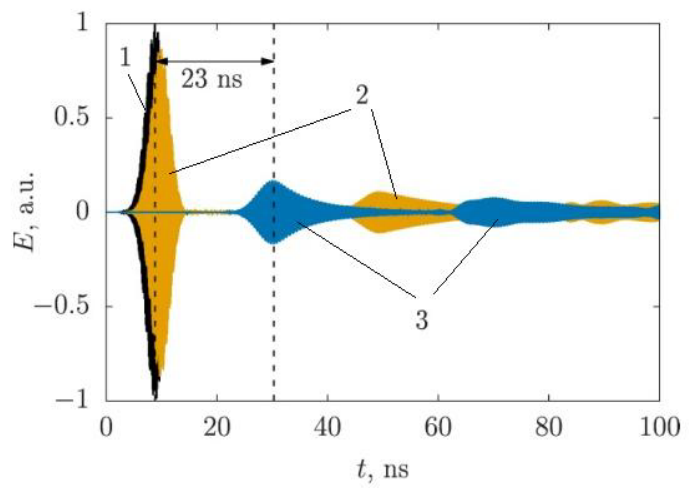

Fig. 2. Simulated electric field strength for the incident (1), reflected (2) and transmitted (3) waves $1.25 \mathrm{~mm}$ diameter placed normally to the wider wave-
guide walls (Fig. 1, a). The crystal period $\mathrm{d}=23.2 \mathrm{~mm}$ corresponds to the half-wavelength in the waveguide for 9.15 GHz frequency. The microwave field structure in the

In the present report the experiment to measure microwave pulse delay in a specially designed photonic crystal (spatially periodic structure built of metal rods inside a WR90/R100 waveguide of $1 \mathrm{~m}$ length) is presented. Each crystal plane comprises 3 copper rods of $1.25 \mathrm{~mm}$ diameter placed normally to the wider wave-
guide walls (Fig. $1, a$ ). The crystal period $\mathrm{d}=23.2 \mathrm{~mm}$

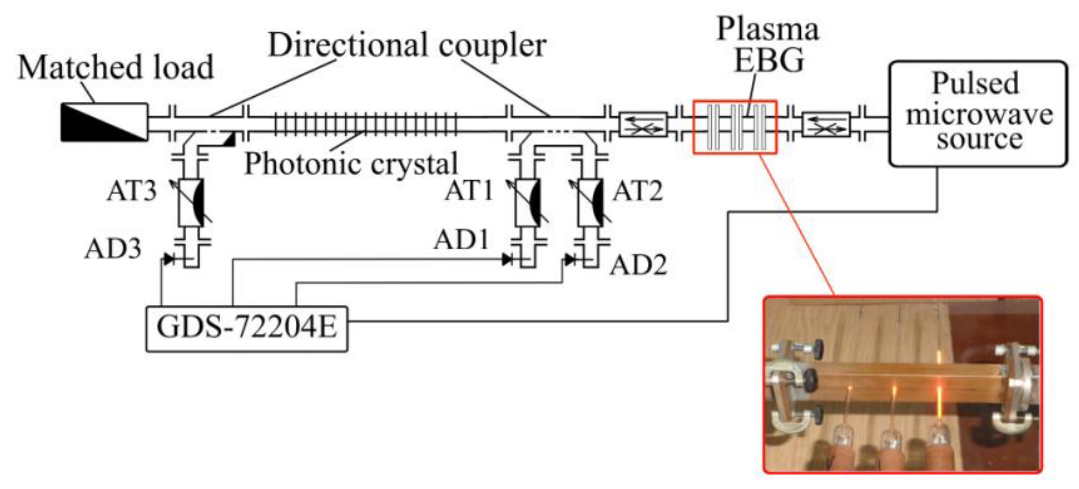

Fig. 3. The experiment layout and the setup photo 
Experiment was produced using a high power magnetron oscillator, which keyword parameters are as follows: frequency about $9.15 \mathrm{GHz}$, pulse duration about $150 \mathrm{~ns}$, repetition rate about $2 \mathrm{kHz}$, peak power $50 \mathrm{~kW}$.

Microwave pulse shortening was produced by a onedimensional electromagnetic band gap (EBG) plasma structure (in other words, electromagnetic crystal) formed in a WR90/R100 waveguide section by 3 discharges in the glass tubes filled by neon at pressure about 70 Torr [6]. These tubes were installed perpendicularly to the wide wall of the waveguide at $30 \mathrm{~mm}$ distance from each other (see Fig. 3, inset at the bottom). When the discharges with electron densities exceeding $10^{14} \mathrm{~cm}^{-3}$ are initiated in the tubes, the plasma electromagnetic crystal, a stop band central frequency of which is about 9.15 $\mathrm{GHz}$, is formed. Discharge ignitions in the tubes take place in result of micro-wave breakdown of working gas under an effect of incident powerful microwave pulses. Incident and transmitted through the plasma electromagnetic crystal pulses are presented at Fig. 4.

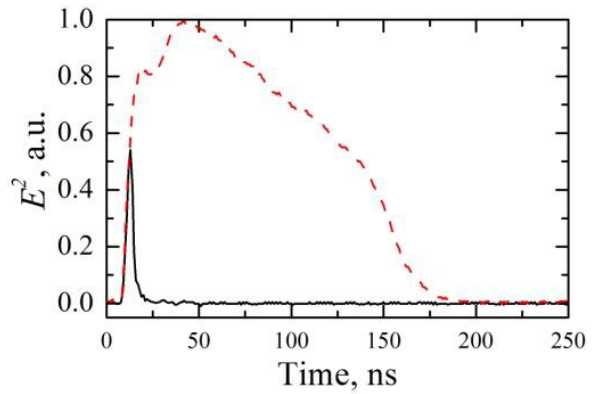

Fig. 4. Microwave pulses incident on the plasma EBG (dashed line) and passed through it (solid line)

Part of the incident to the photonic crystal microwave power was guided by an additional coupler, attenuator AT1 and waveguide-to-coaxial transition AD1 to a microwave diode and detected by oscilloscope GDS72204E (200 MHz, 1 Gigasample/sec). Reflected and transmitted pulses were similarly detected by the same oscilloscope via channels AT2-AD2-microwave diode and AT3-AD3-microwave diode, respectively. Oscilloscope is triggered by sync pulse from the microwave source. To ensure equity of measuring arms the layout was preliminary tested with the empty waveguide of the equal length and without the photonic crystal.

Microwave pulse transmitted through the photonic crystal was normalized to the power of the incident pulse and compared with that obtained in simulation. The detected envelopes of the incident microwave pulses and the transmitted one are shown at Fig. 5 by (line+symbols) curve. The envelope shaded area in Fig. 5 shows simulated squared electric field strength $\left(E^{2}\right)$ for the pulses.

Comparison of experimentally obtained and simulated pulse envelopes demonstrates good agreement in shapes of peaks and their amplitudes. The amplitude of the transmitted pulse is observed to be 30 times smaller as compared to the amplitude of the incident pulse. The observed delay time is $\tau \approx 23 \mathrm{~ns}$ that is also in good agreement with simulation results. The pulse group velocity $v_{g r}$ in the photonic crystal is $v_{g r}=L_{c r} / \tau \approx$ $\approx 3.2 \times 10^{7} \mathrm{~m} / \mathrm{s} \approx 0.11 \mathrm{c}$, where $\mathrm{c}$ is the speed of light in vacuum. Also as it was predicted in simulations the transmitted signal oscillations were detected experimentally. Signal peaks were separated at about 46 ns that in full accordance with the simulation results.
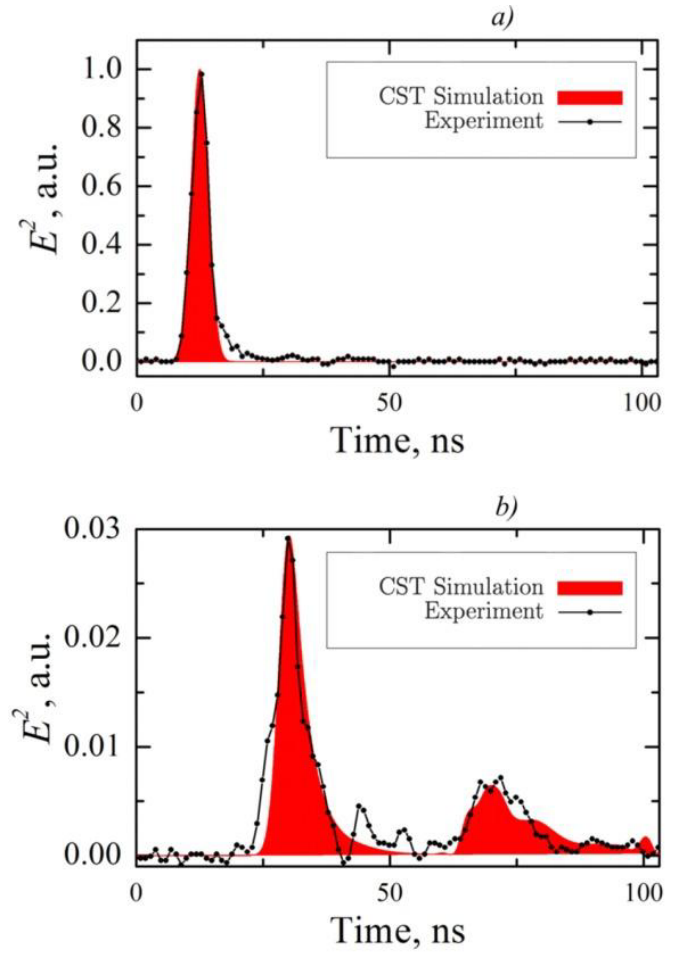

Fig. 5. (a) The envelope of the microwave pulse incident on the photonic crystal and (b) the microwave signal transmitted through it: experiment (line+symbols curve), simulation (shaded area)

The obtained results can be applied in high-speed information transmission systems, which use periodic crystal-like structures to control propagation of microwave radiation.

\section{References}

1. Aleksandrov E.B., Zapasskii V.S. // Phys. Usp. 2006. V. 49. 10671075.

2. Voronin V.V., Lapin E.G. et al. // JETP Letters. 2000. V. 71. P. 76.

3. Baba T. // Nature Photonics. 2008. V. 2. P. 465. 2011.

4. Baryshevsky V.G., Gurinovich A.A. // arXiv:1101.4162.

5. https://www.cst.com/products/cstmws

6. Arkhipenko V.I., Callegari Th. et al. // Journal of Applied Physics. 2014. V. 116. 123302. 